\title{
Kynurenines and the nervous system: therapeutic perspectives
}

\author{
László Vécsei
}

Published online: 11 December 2011

(C) Springer-Verlag 2011

L-tryptophan is involved in protein synthesis and acts as a precursor of many biologically active substances. Besides its participation in the process of protein synthesis, in mammals, tryptophan is metabolised in several pathways. The most commonly known is the serotonergic pathway, which is active in platelets and neurons and yields 5-hydroxy-tryptophan and then serotonin. Tryptophan is also the precursor of a pineal hormone, melatonin. A less well-known, but actually the main alternative route for tryptophan metabolism, is through the kynurenine pathway. It is noticeable that $95 \%$ of tryptophan is catabolised through the kynurenine pathway within the brain. Among these substances, kynurenic acid has potential neuroprotective action blocking glutamate release and glutamate neurotransmission.

The first indication that kynurenines might play a role in different brain functions was provided by Lapin (1978). However, kynurenic acid was detected in the canine urine as early as 1853 (Liebig, 1853).

The discovery of the importance of kynurenines in brain function under physiological and pathologic conditions has led to the identification of potential new drug targets exploiting the therapeutic potential of the pathway. Some of these compounds proved to provide neuroprotection in animal models of various human diseases, which holds promise that their effectiveness well translate to the clinic in the future. Furthermore, the fluctuations in the levels of the kynurenine metabolites have discrete effects on the immune system.

The present series of papers focuses on synthesis of kynurenic acid analogues, nanoscale containers for biomedical application in drug delivery, preclinical behavioural and pharmacological studies and some clinical aspects (headache, Huntington's disease, Parkinson's disease, stroke, multiple sclerosis, amyotrophic lateral sclerosis, major depression, anhedonia). Based on our recent findings the Hungarian Academy of Sciences decided to support a Neuroscience Research Group at the University of Szeged. Thanks are due to the authors of the papers for their excellent contributions.

\footnotetext{
L. Vécsei

Department of Neurology, Faculty of Medicine Albert

Szent-Györgyi Clinical Center, University of Szeged,

Semmelweis u. 6, Szeged 6725, Hungary

L. Vécsei $(\bowtie)$

P.O.Box 427, Szeged 6701, Hungary

e-mail: vecsei.laszlo@med.u-szeged.hu

URL: http://www.szote.u-szeged.hu/medcentrum/neur/
} 